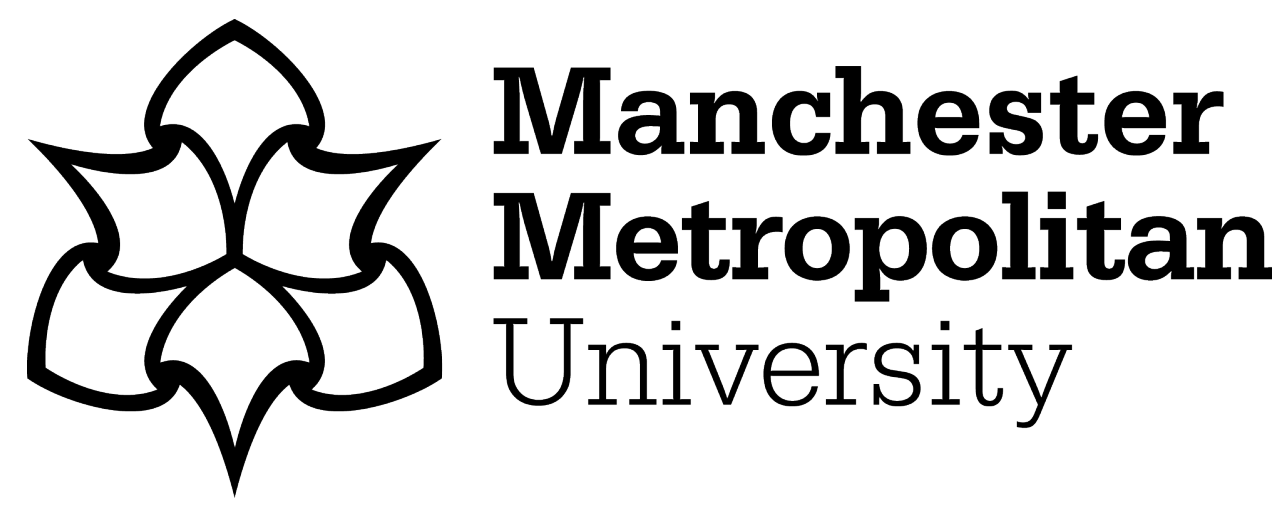

Seyedolhosseini, Atefesadat, Masoumi, Nasser, Modarressi, Mehdi and Karimian, Noushin (2019) Illumination Control of Smart Indoor Lighting Systems Consists of Multiple Zones. In: 2018 Smart Grid Conference (SGC), 28 November 2018 - 29 November 2018, Sanandaj, Iran.

Downloaded from: https://e-space.mmu.ac.uk/626762/

Publisher: IEEE

DOI: https://doi.org/10.1109/sgc.2018.8777883

Please cite the published version 


\section{Illumination Control of Smart Indoor Lighting Systems Consists of Multiple Zones}

\author{
Atefesadat Seyedolhosseini \\ CST-LAB, Department of Electronics \\ School of Electrical and Computer \\ Engineering, College of Eng. \\ University of Tehran, Tehran, Iran
}

\author{
Nasser Masoumi \\ CST-LAB, Department of electronics \\ School of Electrical and Computer \\ Engineering, College of Eng. \\ University of Tehran, Tehran, Iran
}

\author{
Mehdi Modarressi \\ Department of Computer, School of \\ Electrical and Computer \\ Engineering, College of Eng., \\ University of Tehran, Tehran, Iran
}

\author{
Noushin Karimian \\ School of Engineering Science, \\ College of Eng., \\ University of Tehran, \\ Tehran, Iran
}

\begin{abstract}
Accurate and power efficient determination of luminaires dimming level is a challenging issue in smart indoor lighting systems, since the lighting system is nonlinear and time variant. In this paper, a smart and power efficient control method is developed in order to determine luminaires dimming level in an indoor environment with multiple work zones. A positive point of the proposed control method is that photodetectors are placed at the work zones which increase the accuracy. Besides, different number of photodetectors can be placed at work zones in the proposed control method, since work zones may have different dimensions and also accuracy levels may differ. The control method takes the advantages of learning method to avoid complexity and also increase system reliability. The system can properly work with daylight variation during the daytime. Case studies are implemented in DIALux and the control method is evaluated in MATLAB. It is shown that the error for static condition is below $1 \%$ and for dynamic condition which daylight varies during daytime is increases to $5.6 \%$.
\end{abstract}

Index Terms - indoor, lighting, smart, neural network, work zone, visual task.

\section{INTRODUCTION}

More intelligent smart environments are resulted from advancements in Sensing, computation, and communication. Smart lighting as major subset of smart environment is witnessing large amount of advances these days, since, artificial lighting system plays an undeniable role in the amount of consumed energy, resident comfort, health, and security.

Major fraction of global electrical energy consumption is mostly dedicated to artificial lighting. In office buildings, the amount of energy consumption due to the artificial lighting can be more than $40 \%$ of the whole energy consumption [1]. Efficient indoor illumination control while maintaining visual comfort is an important issue which also can yield to the lower system cost [2]. The main focus of this paper is illumination control of indoor environments.

Sensors (photodetector and presence detector), dimmable luminaires such as light emitting diodes (LEDs), and controller are indoor lighting system elements which affect system performance [3]-[5]. Each indoor environment may be partitioned into several work areas and a specific visual task is carried out in each work zone [6]. The main problem in lighting systems is to adjust dimming level of luminaires such that maintaining visual comfort without increase in energy consumption. Luminaire's dimming levels are determined by the control algorithm applied into the controller and the decisions are made by the visual task carried out in the work zone, occupancy condition, and current intensities measured by the sensors [7]-[9].

There are various control methods such as centralized, decentralized and distributed, which each has pros and cons in system efficiency, performance, and power consumption [9]-[13]. Light intensity is measured as Lux $(l x)$ at each point and lighting uniformity is defined as the smoothness of lighting distribution across the work zone.

Some research have been done in order to efficiently determine luminaires dimming level [8]-[13]. Researchers model the photodetector's output as a function of luminaires dimming level, daylight, and sensor's noise which is known by dark current in [8]. The authors in [8] assume linear relation between sensor's output and luminaire's dimming level. Luminaire's effect on each sensor is modeled by light transport matrix which theoretically cannot be computed [9]. Thus, optimization problems are used in order to determine dimming levels. In the work done in [9], a region is extracted for matrix argument which by that, presented control loop is stable and it is determined by Gershgorin's circle theorem. Multiple control steps are needed to minimize error to a desired area each time that lighting condition varies. Therefore, the designed system is not optimized especially in wireless sensor networks (WSNs) in on-battery nodes. A method in [10] is presented based on the methods applied in [9]. In this work number of photodetectors are equal to the number luminaires owing to analytical limitations. However, indoor environments can be divided into several work zones and each may have multiple photodetectors.

Some other researches according to the idea that lighting system is non-linear and time variant with various types of 
luminaires proposed control systems to adjust the dimming level of light sources [11]-[13]. A control loop has been designed in order to adjust dimming level of luminaires with different wavelength [11]; where a crucial parameter of zoning effect has not been taken into account, and no information on the daylight effect on system performance has been reported. In a separate study, learning methods is applied in order to develop a smart lighting system and the method is applicable for static condition where daylight does not vary during daytime and objects are constant [12]. Besides, transmission is not considered in the discussed methods which is an important parameter [14].

In this paper, an indoor environment with multiple work zones that different visual task can be carried out in each and also variant occupancy condition is considered to design control method. Besides, each zone may have various number of photodetectors which makes theoretical aspects useless in this area of research. The system is also considered dynamic due to the daylight variation. The method proposed in this paper accurately determines luminaire's dimming level according to the standard requirements and also with low transmission rate.

\section{LEARNING CAPABLE CONTROL METHOD}

The control method is a major subset of indoor smart lighting system. The control algorithm which is applied in the controller based on the system inputs determines the dimming level of luminaires (output), as shown in Figure 1. The indoor environment is divided into $q$ work zones; thus, the system is equipped with $q$ presence detectors. The outputs of presence detectors are applied as inputs to the controller $\left(O C_{q}\right)$. The average value of light intensity and lighting uniformity at $i^{\text {th }}$ zone should be greater than $E_{m}$ and $U_{m}$, which is determined by the standard [6]. The controller uses the measured intensity of $p$ placed photodetectors as feedback to determine dimming level of all $r$ luminaires. Inputs and outputs in the lighting system is transferred by the communication modules which mostly are wireless in indoor smart environments [1] and the communication issues are beyond the scope of this paper.

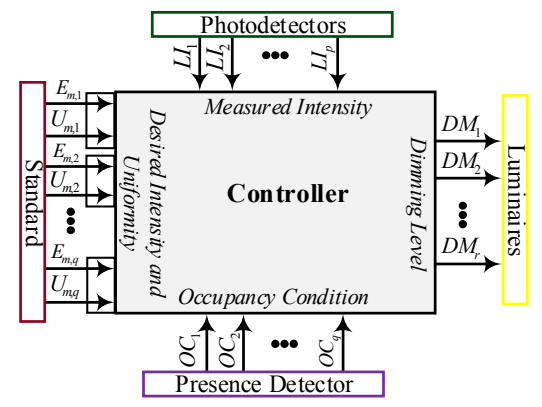

Figure 1. The controller inputs and outputs as a subset of indoor smart lighting system

In the system that $q \neq p \neq r$ theoretical approaches are not applicable any more, each zone occupancy affects other zone illumination condition due the reflections caused by object surfaces [1]. Thus, optimization methods may be considered as a useful solution to calculate the luminaire's dimming level [12], [13]. The methods with learning capability are one of these. The learning methods is trained once and work with less data transmission through the network in static condition, since most of smart lighting systems are based on wireless communication protocols and in such systems transmission rate is an important parameter due to the power consumption. In this paper, daylight variation during the daytime is also considered in order to reduce power consumption and increase the accuracy of the system.

The proposed controller in this paper has three main working modes, as shown in Figure 2. In this paper, we have assumed that the controller has knowledge about the system elements; number of zones, photodetectors, luminaires, and driver dimming steps.

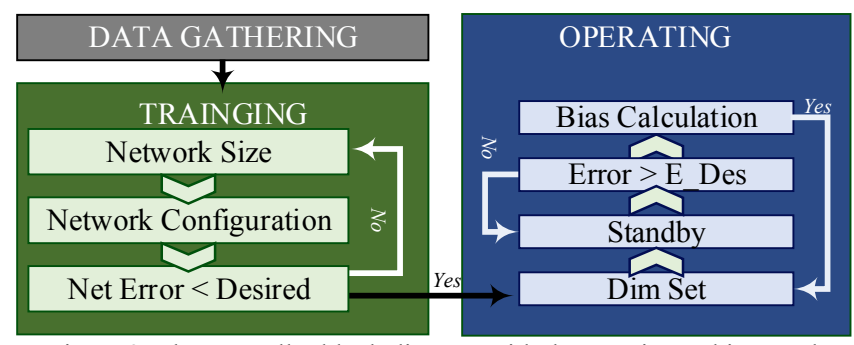

Figure 2. The controller block diagram with three main working modes

The first mode in the proposed controller is DATA GATHERING as shown in gray box in Figure 2. In this state, controller request luminaires to dim one by one and in groups and after each step controller waits until the photodetector modules sends back the measured intensities. Therefore, a loop up table is formed in the controller. This mode can be done in an offline mode; therefore, on-battery nodes in Wireless Sensor Networks (WSNs) is not adversely affected due to the extra power consumption. After finalizing this step, the controller changes its state to TRAINING mode. The neural network is configured in this state. Feedforward neural network is considered for the proposed method while other learning methods also can be applied. The minimum neuron count is considered for the network size, since increase in number of neurons results in higher computational power [12]. While network error is beyond the desired value, the controller increase the neuron counts, as shown in Figure 2. The neural network inputs are $E_{m}$ and $U_{m}$. Thus, the reported intensities from the photodetectors to the controller is used to compute these values.

$$
\begin{gathered}
\text { meas_- } E_{m, i}=\frac{\sum_{j=1}^{n} L I_{j}}{n} \\
\text { meas_ } U_{m, i}=\frac{\min \left(L I_{i, 1}, L I_{i, 2}, \ldots, L I_{i, n}\right)}{E_{m, i}}
\end{gathered}
$$

where in (1) and (2), the parameter $n$ represents the number of photodetectors at the $i^{\text {th }}$ zone. Average light intensity (meas $E_{m}$ ) and lighting uniformity (meas_ $\left.U_{m}\right)$ are calculated at a pre-block to the neural network block as inputs to it. Average light intensity and lighting uniformity is also calculated for all zones.

After the network is successfully configured, the controller changes its state to the OPERATIONAL mode. Initially, luminaires dimming level is set. For the zones with no occupancy desired average intensity and uniformity is considered the current value of the photodectors. As dimming level of the luminaires are determined the controller moves to the standby. After 30 minutes, the controller checks the error. The average light intensity and lighting uniformity of each zone extracted from the sensors are compared while the uniformity and average intensity is beyond desired values controller goes back to the standby state. The bias 
which is difference between desired and measured values is calculated when the measured values are lower than desired ones.

$$
\begin{gathered}
\text { bias_ } E_{m, i}=E_{m, i}-\text { Meas_ } E_{m, i} \\
E_{m, i}=E_{m, i}+b i a s_{-} E_{m, i}
\end{gathered}
$$

The bias_ $E_{m, i}$ for the $i^{\text {th }}$ zone is calculated through (3) and is added to desired value as in (4) and new desired values is calculated and it is considered as new inputs to the neural network block. It worth noting that the values computed in (3) and (4) is for the condition that the measured values for $i^{\text {th }}$ zones are below the desired values.

In the proposed novel controller, in this paper, dynamic conditions such daylight variation and system element faults are taken into account by adding a bias to desired average intensity and lighting uniformity in order to improve the accuracy. The controller can be applied for different indoor environments with multiple number of zones and sensors.

\section{CASE StUdiES AND SimUlation RESUlts}

The controller proposed in this paper is suitable for indoor environments that have multiple works zones, various number of photodetectors on each zone of environment, and during the daytime photodetectors outputs varies.

Two test cases are implemented in DIALux in order to evaluate the proposed control mechanism. The $3 \mathrm{~d}$ view of both rooms is shown in Figure 3; as shown each room has window that enters daylight in the environment. Each desk is considered as a single work zone and the zone numbers of are labeled on desks in Figure 3. Each has physical parameters which in detail are introduced in TABLE I. Zones with unequal number of sensors are considered for the case (a) to investigate the effect of sensor variations; furthermore, number of luminaires are not equal to the number of zones and also photodetectors. Three work zones are placed in Case (b) that each receives different daylight effect.

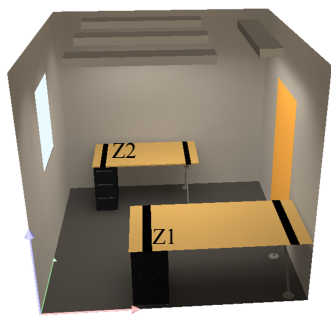

(a)

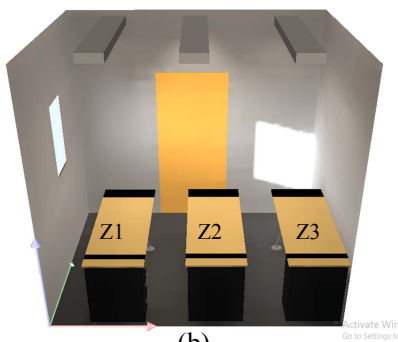

(b)
Figure 3. 3D view of two test cases with different specifications

As mentioned in previous section, the network is initially configured with minimum size (2 neurons) and its size is increased while the network error is in the desired region. Error below $1 \%$ is desirable for the test cases introduced in Figure 3 and TABLE I. As Mentioned Earlier, the feedforward neural network is considered for configuration as shown in Figure 4. The number network inputs are equal to the number of zones multiplied by two; average light intensity and lighting uniformity are two parameter considered as inputs for each zone. The network outputs are dimming level of luminaires and thus the network outputs layer size is equal to the number of luminaires. The hidden layer size is determined during the training mode and it is considered as $t$ in Figure 4.

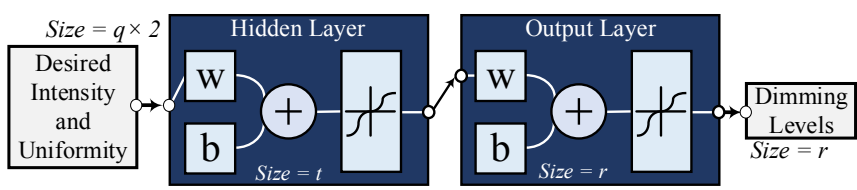

Figure 4 . The neural network block with $2 \mathrm{q}$ inputs, $\mathrm{r}$ output layers which is equal to number of luminaires, and thidden layers that is determined during network configuration

Neural network for Case (a) in Figure 1 is configured for different hidden layer sizes with 1096 datasets for training states. The network performance is evaluated for 500 datasets for each network sizes, as shown in Figure 5. The average intensity and uniformity for each test set is calculated and it is compared to the desired value, if the desired value is smaller than the measured the error is considered as 0 . The uniformity mentioned in standard is the lowest and higher uniformity value is also more desired [6]. If the desired value of average intensity or lighting uniformity is lower than desired values, the error is recorded in percent. The average calculated error of 500 cases for each zone of case (a) is shown in Figure 5. As shown in Figure the error is below 1\% for both zones. The error histogram of neural network for 1096 data for training with network size equal to 5 is shown in Figure 6.

The error histogram of case (b) in Figure 3, is shown in Figure 7. 115 datasets is used to train the network and the network size of 6 is selected after training status, shown in Figure 7. The network error for both average light intensity and lighting uniformity of all three zones of case (b) is reported in TABLE II. As it is reported, the network error for all zones in the case is also converged to desired accuracy.

In the last step, daylight variation is also added to the system, since in real systems daytime variation should be taken into account. Daylight effect is considered as bias to the system as introduced in (3) and (4). The maximum error for 24 test sets in different time of day is $5.6 \%$ for case (a) and for the case (b) it is reduced to $3.8 \%$.

Efficient and accurate control system in order to adjust luminaires dimming level in smart lighting system has been introduced in this section. As the simulation results is shown the proposed controller maximum error is 5.6\% and for static condition (without the daylight variation) it is below $1 \%$ with optimum number of data transfer through the network.

TABLE I. Physical Parameters of Implemented Test CASES IN DialuX

\begin{tabular}{|c|c|c|c|c|c|c|c|c|c|c|}
\hline Case & $\begin{array}{c}\text { Room } \\
\text { Area } \\
\left(\mathrm{m}^{2}\right)\end{array}$ & $\begin{array}{c}\text { Room } \\
\text { Height } \\
(\mathrm{m})\end{array}$ & $\begin{array}{c}\text { Wall } \\
\text { reflection } \\
(\%)\end{array}$ & $\begin{array}{c}\text { Number } \\
\text { of Zones }\end{array}$ & $\begin{array}{c}\text { Number of } \\
\text { Luminaires }\end{array}$ & $\begin{array}{c}\text { Work zones } \\
\text { area } \\
\left(\mathrm{m}^{2}\right)\end{array}$ & $\begin{array}{c}\text { Work zones } \\
\text { height }(\mathrm{m})\end{array}$ & $\begin{array}{c}\text { Number of } \\
\text { sensors per } \\
\text { zone }\end{array}$ & $\begin{array}{c}\text { Avg. Daylight } \\
\text { deviation* }\end{array}$ & $\begin{array}{c}\text { Sky } \\
\text { Type }\end{array}$ \\
\hline $\mathrm{a}$ & 9 & 2.74 & 30 & 2 & 4 & $\begin{array}{c}\mathrm{Z} 1=1.5 \times 0.8 \\
\mathrm{Z} 2=2 \times 1\end{array}$ & 0.76 & $\begin{array}{c}\mathrm{Z} 1=9 \\
\mathrm{Z} 2=13\end{array}$ & $\begin{array}{c}\mathrm{Z} 1=83 \% \\
\mathrm{Z} 2=49 \%\end{array}$ & $\mathrm{Clear}$ \\
\hline $\mathrm{b}$ & 6.6 & 2.74 & 40 & 3 & 3 & $1.5 \times 0.6$ & 0.76 & 3 & $\mathrm{Z} 1=57 \%, \mathrm{Z} 2=64 \%$ & $\mathrm{Clear}$ \\
\hline
\end{tabular}




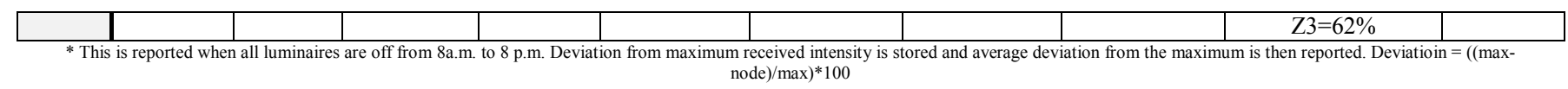

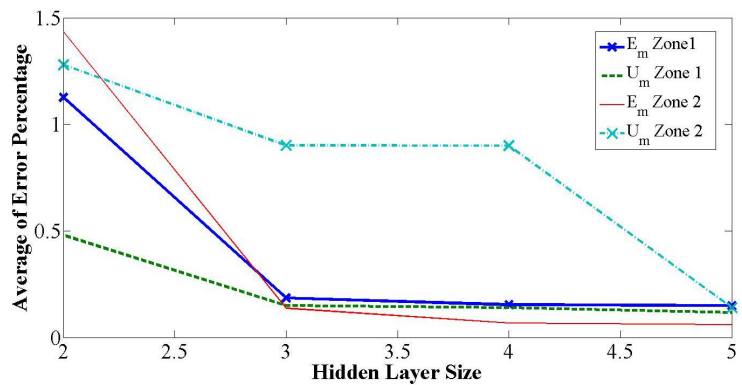

Figure 5. The average percentage error between desired and measured value of average intensity and lighting uniformity in case (a) and two zones in variation of hidden layer size from 2 neurons to 5 for 500 datasets

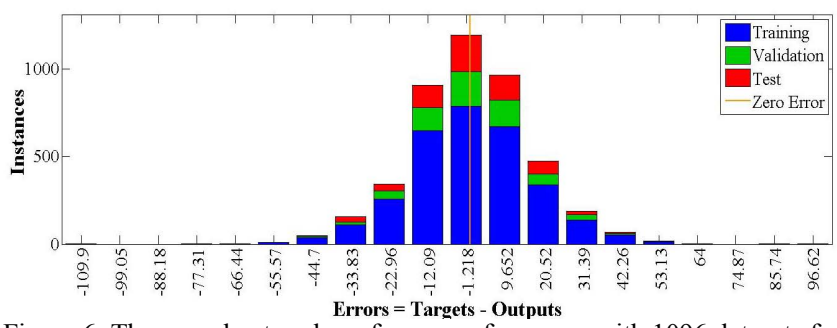

Figure 6. The neural network performance for case a with 1096 datasets for training and 5 neurons for hidden layer, the network has 4 inputs and 4 outputs

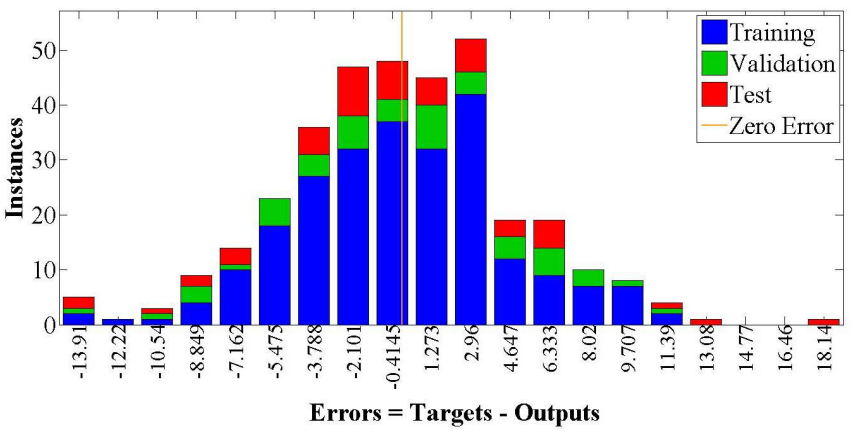

Figure 7. The neural network performance for case (b) with 115 datasets for training and 6 neurons for hidden layer, the network has 6 inputs and 3 outputs

TABLE II. Average of Error Percentage Between Desired and Measured Values for 40 Test Data of CASE (B)

\begin{tabular}{|c|c|c|c|c|c|}
\hline \multirow{2}{*}{ Par. } & \multicolumn{5}{|c|}{ Average of Error percentage vs. Number of Hidden } \\
\cline { 2 - 6 } & 2 & 3 & 4 & 5 & 6 \\
\hline$E_{m, 1}$ & 2.15 & 1.82 & 1.00 & 0.66 & 0.36 \\
\hline$U_{m, 1}$ & 1.65 & 1.01 & 0.68 & 0.13 & 0.01 \\
\hline$E_{m .2}$ & 1.65 & 0.98 & 0.64 & 0.54 & 0.24 \\
\hline$U_{m .2}$ & 1.41 & 0.42 & 0.32 & 0.14 & 0.01 \\
\hline$E_{m .3}$ & 1.63 & 0.96 & 0.58 & 0.43 & 0.24 \\
\hline$U_{m .3}$ & 1.64 & 0.51 & 0.28 & 0.11 & 0.01 \\
\hline
\end{tabular}

IV. CONCLUSION

Illumination control of indoor lighting offices as an important issue is investigated in this paper. The control method suitable for indoor environments consisting of multiple work zones is presented. In the proposed method control method, different zones can have unequal number of photodetectors without increase in complexity. Besides, photodetectors are placed at the work zones which results in improvement of accuracy and also it reduces extra power consumption compared to ones that photodetectors are placed adjacent to the luminaires. The daylight variation during daytime in the office area is also taken into account by the bias calculation process. The method is evaluated by linking DIALux and MATLAB. The results shown error below $1 \%$ for static condition. In case of daylight variation, the maximum error is $5.6 \%$ for investigated test cases.

\section{ACKNOWLEDGMENT}

This work was supported by RF Circuit and Systems Design and Test Lab. and Advanced VLSI Laboratory

\section{REFERENCES}

[1] A. Pandharipande and D. Caicedo, "Smart Indoor Lighting Systems with Luminaire-Based Sensing: A Review of Lighting Control Approaches," Elsevier Journal on Energy and Buildings, no. 104, pp. 369-377, 2015.

[2] H. Zou, Y. Zhou, H. Jiang, S. Ch. Chien, L. Xie, C. J. Spanos, "WinLight: A WiFi-Based Occupancy-Driven Lighting Control System for Smart Building," Elsevier Journal on Energy and Buildings, no. 158 , pp. 924-938, 2018

[3] M. Beccali, M. Bonomolo, G. Ciulla, and V. L. Brano, "Assessement of Indoor Illuminance and Study on Best Photosensors position for Design and Commissioning of Daylight Linked Control Systems. A New Method Based on Artificial Neural Networks," Elsevier Journal on Energy, no. 154, pp. 466-476, 2018.

[4] A. Panharipande, G. R. Newsham, "Lighting Controls: Evolution and Revolusion," Lighting Reseasrch \& Technology, no. 50, pp. 115-128, 2018.

[5] A. Pandharipand and D. Caicedo, "Daylight Illumination Control of LED Systems Based on Enhanced Presence Sensing" Elsevier Journal on Energy and Buildings, no. 43, pp. 944-950, 2011.

[6] Light and Lighting - Lighting of Work Places - Part I: Indoor Work Places, European Standard, EN 12464-1, 2011.

[7] P. Ihm, A. Nemri, and M. Krarti, "Estimation of Lighting Energy Saving from Daylighing," Elsevier Journal on Energy and Buildings, no. 44, pp. 509-514, 2009.

[8] D. Caicedo and A. Pandharipande, "Distributed Illumination Control with Local Sensing and Actuation in Networked Lighting Systems," IEEE Sensors Journal, vol. 13, no. 3, March 2013.

[9] S. Afshari, S. Mishra, A. Julius, F. Lizarralde, J. D. Wason, J. T. Wen, "Modeling and Control of Color Tunable Lighting Systems," Elsevier Journal on Energy and Buildings, no. 68, pp. 242-253, 2014.

[10] A. Afshari and S. Mishra, "A Plug-and-Play Realization of Decentralized Feedback Control for Smart Lighting Systems," IEEE Trans. On Control Systems Technology, vol. 24, no. 4, July 2016.

[11] I. Chew, V. Kalavally, Ch. P. Tan, and J. Parkkinen, "A SpectrallyTunable Smart LED Lighting System with Closed-Loop Control," IEEE Sensors Journal, vol. 16, no. 11, 2016.

[12] D. Tran and Y. Kh. Tan, "Sensorless Illumination Control of Networked LED-Lighting System Using Feedforward Neural Network," IEEE Trans. On Industrial Electronics, vol. 61, no. 4, Apr. 2014.

[13] Sh. Li, A. Pandharipand, and F. M. J. Willems, "Daylight Sensing LED Lighting System," IEEE Sensors Journal, vol. 16, no. 9, May 2016.

[14] A. Seyedolhosseini, N. Masoumi, and M. Modaressi, "Performance Improvement of ZigBee Networks in Coexitence of Wi-Fi Signals." The 7 th International Conference on Information Communication and Management (ICICM), Mosco, Russia, 2017. 